\title{
Enhanced expression of bone morphogenetic protein system in aldosterone-treated mouse kidneys
}

\author{
Jiro Suzuki, Fumio Otsuka, Yoshinori Matsumoto, Kenichi Inagaki, Tomoko Miyoshi, Masaya Takeda, \\ Naoko Tsukamoto, Eri Nakamura, Kanako Ogura and Hirofumi Makino
}

\begin{abstract}
Recent studies have shown that bone morphogenetic proteins (BMPs), particularly BMP-7, have an inhibitory role in the development of various renal diseases. We previously reported antagonistic effects of BMPs on renal mesangial cell proliferation induced by aldosterone (Aldo) in vitro. In the present study, we investigated in vivo roles of BMPs in Aldo-induced renal glomerular injury. BALB/c mice aged 6 weeks were treated with Aldo injection ( $5 \mu \mathrm{g}$ per day, intraperitoneally) and/or oral administration of high-salt (2\%) water for 9 weeks. Systemic blood pressure, body weight, kidney weight and daily proteinuria were not significantly changed by Aldo and/or high-salt treatment. However, renal histological examination revealed increases in glomerular cellularity and glomerular diameter in the groups treated with Aldo injection and high-salt administration. Immunohistochemistry demonstrated expression of BMP-4 and -7 in the glomerular mesangial region. Aldo causes renal glomerular damage by stimulating mesangial cell proliferation and increasing extracellular matrix via the mineralocorticoid receptor (MR). MR messenger RNA (mRNA) expression in the renal cortex was transiently increased by 3-week treatment with Aldo and high-salt intake, but was decreased by 9-week treatment. Furthermore, the expression levels of BMP-4 and -7 mRNA were enhanced in the renal cortex treated with Aldo and high-salt administration. These findings suggest that the renal BMP system is activated by Aldo under the condition of high-salt exposure, which may have a key role in antagonizing glomerular damage in vivo.
\end{abstract}

Hypertension Research (2012) 35, 312-317; doi:10.1038/hr.2011.186; published online 10 November 2011

Keywords: aldosterone; bone morphogenetic protein (BMP); glomerulus; kidney; salt-sensitive hypertension

\section{INTRODUCTION}

Aldosterone (Aldo) has been shown to be a risk factor for multiple organ damage, including damage to the cardiovascular system, brain and kidney, in addition to its regulatory role in body electrolytes and water homeostasis. Recent studies have shown that Aldo directly induces renal glomerular damage by increasing the extracellular matrix and by stimulating mesangial cell proliferation. ${ }^{1,2}$

Mesangial cell proliferation is a key parameter of glomerular damage attributed to the effects of Aldo. In vivo studies have shown that administration of Aldo and salt resulted in glomerular injury characterized in rat kidneys by mesangial matrix expansion and mesangial cell proliferation. ${ }^{3}$ Activity of mitogen-activated protein kinase (MAPK) pathway in renal cortical tissue $e^{3}$ and mesangial cells ${ }^{4}$ via the mineralocorticoid receptor (MR) is functionally involved in the Aldo-induced mesangial cell proliferation. MR expression is detected in renal glomeruli, although the expression level is lower than in the distal tubular epithelium. ${ }^{5}$ Given that glomerular MR is confined to mesangial cells, the density of MR expression could be substantially the same as that in the distal epithelium. ${ }^{6}$ Considering that mesangial proliferation and deformity induced by Aldo are antagonized by eplerenone, ${ }^{4}$ the MR may have a key physiological role in maintaining mesangial morphology in the glomeruli.

Bone morphogenetic proteins (BMPs), which belong to the transforming growth factor (TGF)- $\beta$ superfamily, have been shown to be important molecules involved in renal differentiation. ${ }^{7}$ Recent studies have provided evidence that BMPs, particularly BMP-7, have a key role in the pathogenesis of various renal diseases including early experimental diabetic ${ }^{8}$ or obstructive ${ }^{9}$ nephropathy. Among BMP ligands, BMP-7 has pivotal roles during embryogenic renal and eye development, ${ }^{10}$ and BMP-7 expression is retained in only a few tissues in adults, most prominently in the kidney. ${ }^{11}$ In experimental animal nephropathy, BMP-7 has been known to ameliorate fibrotic changes of renal tissues by antagonizing TGF- $\beta$-induced profibrogenic activities. $^{12,13}$ It is also reported that BMP-4 and BMP-7 are highly expressed in prospective podocytes and parietal epithelial cells of developing glomeruli, suggesting that BMP may have a role in glomerulogenesis. ${ }^{14}$

We previously reported antagonistic effects of BMPs on renal mesangial cell proliferation induced by Aldo in vitro. ${ }^{15}$ It was revealed that BMP-4 and BMP-7 specifically antagonized Aldo-induced mesan- 
gial cell proliferation through inhibition of MAPK activation and MR expression. ${ }^{15}$ In the present study, we investigated in vivo changes in the renal BMP system molecules in Aldo-induced renal glomerular injury.

\section{METHODS}

\section{Animal procedure}

BALB/c male mice aged 6 weeks were purchased from Japan SLC (Shizuoka, Japan). All mice were housed in climate-controlled metabolic cages with a 12:12-h light-dark cycle. The animals received normal food containing $0.24 \%$ sodium (MF, Oriental Yeast, Tokyo, Japan), with water provided ad libitum. Mice were treated with Aldo injection $\left(5 \mathrm{mg} \mathrm{ml}^{-1}\right.$ per day, intraperitoneally) and/or oral administration of $2 \% \mathrm{NaCl}$-containing water for 9 weeks. Control mice were treated with phosphate-buffered saline (PBS) injection ( $1 \mathrm{ml}$ per day, intraperitoneally) and/or oral administration of distilled water for 9 weeks. Systolic blood pressure, measured by tail-cuff method, and body weight (BW) were continuously monitored. Excretion of urinary protein for $24 \mathrm{~h}$ was measured (Bio-Rad protein assay kit, Bio-Rad, Richmond, CA, USA) at indicated time points after the commencement of each treatment. After indicated treatment for 3 weeks and 9 weeks, mice were killed and specimens of the renal cortex were analyzed. The experimental protocol was approved by the Ethics Review Committee for Animal Experimentation of Okayama University Medical School.

\section{Histological analysis}

For study of glomerular histology, a portion of each kidney was fixed in $10 \%$ buffered paraformaldehyde, embedded in paraffin, sectioned at $4 \mu \mathrm{m}$ and stained with hematoxylin-eosin. Histological examination of glomeruli was microscopically performed as we previously reported. ${ }^{16}$ Histologic assessment included measurement of the diameter of each of 200 randomly selected glomeruli in each experimental group. The total number of cells containing nuclei in each glomerulus of at least 100 glomeruli in each specimen was counted. The diameters $(\mu \mathrm{m})$ of 200 randomly selected glomeruli in each experimental group were measured using an objective micrometer (Olympus Optical, Tokyo, Japan), and histograms of glomerular cross-sections were compared among the four groups.

\section{Immunohistochemical study}

For immunohistochemical analyses, $4-\mu \mathrm{m}$-thick sections of formalin-fixed and paraffin-embedded renal tissues were dewaxed and rehydrated, and antigen retrieval was performed by heating for $10 \mathrm{~min}$ in $10 \mathrm{~mm}$ citrate buffer at $\mathrm{pH}$ 6.0. The sections were reacted with goat polyclonal antibodies for BMP-4 and BMP7 (R\&D Systems, Minneapolis, MN, USA), and a normal goat control antibody (R\&D Systems) diluted at $15 \mu \mathrm{g} \mathrm{ml}^{-1}$ for $16 \mathrm{~h}$ at $4{ }^{\circ} \mathrm{C}$, and were then stained using the universal immuno-peroxidase polymer method with a Histofine staining kit (Nichirei Corp., Tokyo, Japan) according to the manufacturer's protocol. Positive reaction was visualized with $3,3^{\prime}$-diaminobenzidine tetrahydrochloride, followed by counterstaining with hematoxylin. For negative controls, a normal goat antibody was used instead of primary antibodies, and no specific immunoreactivity was detected in these sections.

\section{RNA extraction, reverse transcriptase PCR and quantitative real-time PCR analysis}

Total RNA of renal cortex tissues from adult male BALB/c mouse kidneys was extracted using TRIzol. Expression of BMP ligands, BMP receptors and MR messenger RNAs (mRNAs) was detected by reverse transcriptase PCR analysis. The extracted RNA $(1.0 \mu \mathrm{g})$ was subjected to an RT reaction using a FirstStrand cDNA synthesis system (Invitrogen Corp., Carlsbad, CA, USA) with random hexamer $\left(2 \mathrm{ng}^{-1}\right)$, reverse transcriptase $(200 \mathrm{U})$ and deoxynucleotide triphosphate $(0.5 \mathrm{~mm})$ at $42^{\circ} \mathrm{C}$ for $50 \mathrm{~min}$ and at $70^{\circ} \mathrm{C}$ for $10 \mathrm{~min}$. Subsequently, hot-start PCR was performed using $\mathrm{MgCl}_{2}$ (1.5 mM), deoxynucleotide triphosphate $(0.2 \mathrm{~mm})$ and $2.5 \mathrm{U}$ of Taq DNA polymerase (Invitrogen Corp.) under the conditions we previously reported..$^{17}$ PCR primer pairs were selected from different exons of the corresponding genes as follows: activin receptor-like kinase (ALK)-2, 639-661 and 1100-1121 (from GenBank accession \#NM_007394); ALK-3, 426-445 and 916-935 (from NM_009758); BMP type
II receptor, 13-32 and 515-534 (from NM_007561); MR, 2978-2999 and 32193240 (from M36074); BMP-4, 200-220 and 420-440 (from NM_007554); BMP-7, 418-438 and 684-704 (from X56906); and a house-keeping gene, ribosomal protein L19 (RPL19), 373-393 and 547-567 (from NM_009078). For the quantification of BMP-4, BMP-7, ALK-2, ALK-3, BMP type II receptor and MR mRNA levels, real-time PCR was performed using a LightCyclerFastStart DNA master SYBR Green I system (Roche Diagnostic Co., Tokyo, Japan) under the condition of annealing at $60-62{ }^{\circ} \mathrm{C}$ with $4 \mathrm{~mm} \mathrm{MgCl}_{2}$ following the manufacturer's protocol. Accumulated levels of fluorescence for each product were analyzed by the second derivative method after meltingcurve analysis (Roche Diagnostic Co.), and then, following assay validation by calculating each amplification efficiency, the expression levels of target genes were quantified on the basis of standard curve analysis for each product. For each transcript, all treatment groups were quantified simultaneously in a single LightCycler run. To correct for differences in RNA quality and quantity between samples, the expression levels of target gene mRNA were normalized by dividing the quantity of the target gene by the quantity of RPL19 in each sample. The raw data of each target mRNA level (/RPL19) were statistically analyzed as indicated and are shown as fold changes in the figures.

\section{Statistical analysis}

All results are shown as means \pm s.e.m. of data from at least three separate experiments, each performed with triplicate samples. The data were subjected to analysis of variance to determine differences and, if differences were detected by analysis of variance, Fisher's protected least significant difference test was used to determine which means differed (StatView 5.0 software, Abacus Concepts, Berkeley, CA, USA). $P$-values $<0.05$ were accepted as statistically significant.

\section{RESULTS}

In vivo effects of Aldo on glomerular damage were investigated using 6-week-old male BALB/c mice. Changes in physiological parameters including systemic blood pressure, BW and kidney weight were determined and compared among the four groups: control group (PBS injection with water intake: $n=6$ ), Aldo group (Aldo injection with water intake: $n=6$ ), $2 \%$ salt group (PBS injection with $2 \%$ salt water intake: $n=6$ ) and Aldo+2\% salt group (Aldo injection with $2 \%$ salt water intake: $n=6$ ). As shown in Figure 1a, time-course changes of systolic blood pressure, BW and kidney weight/BW ratio were not significantly different among the four groups throughout the 9-week experimental period. In addition, 24 -h excretion of urinary protein level was not differed significantly among the four groups during the 9-week experimental period (Figure 1b).

Glomerular changes caused by Aldo in combination with high-salt treatment were histologically examined. As shown in Figure 2a, Aldo treatment with high-salt $(2 \%)$ administration increased glomerular cellularity with increased size of each glomerulus. To characterize the differences among the treated groups, glomerular cellularity and glomerular size were numerically evaluated. As shown in Figure $2 b$, glomerular cellularity score was significantly higher in the group treated with Aldo injection and $2 \%$ salt administration than in the control group at 3 weeks and 9 weeks.

Effects of Aldo and high-salt administration on MR expression in the renal cortex of BALB/c mice are shown in Figure 3. Based on the analysis of quantitative real-time PCR, MR mRNA expression in the renal cortex was transiently increased by 3 -week treatment with Aldo and high salt, whereas MR expression was decreased by 9-week treatment with Aldo injection and high-salt intake. The expression of BMP was examined by immunohistochemical analysis, in which BMP-4 and BMP-7 protein expression was detected in the glomerular mesangial region (Figure 4a). Quantitative PCR analysis showed that mRNA expression of BMP-4 was increased by Aldo injection and high-salt intake for 3 to 9 weeks and that mRNA expression of BMP-7 was also upregulated by 9 -week treatment with Aldo and high-salt intake (Figure $4 \mathrm{~b}$ ). 
a

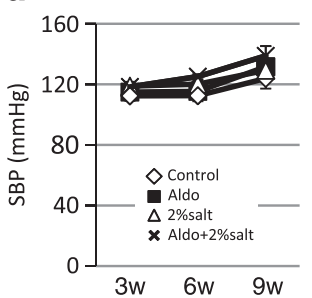

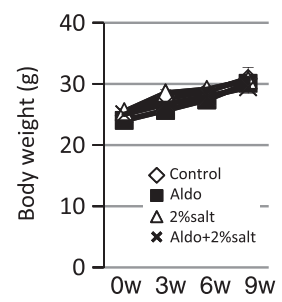
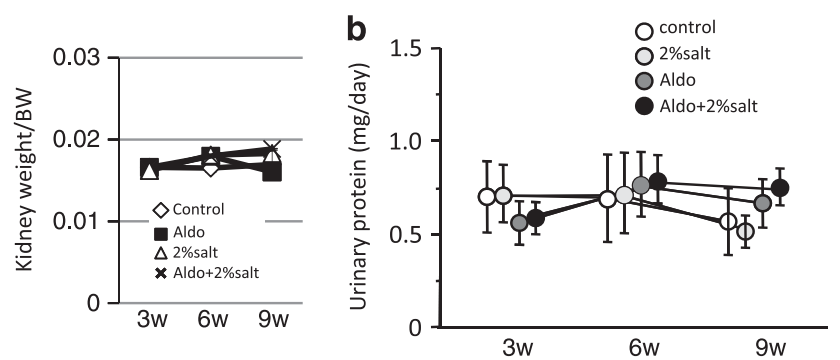

Figure 1 Changes in physiological parameters caused by Aldo and high-salt treatment. BALB/c male mice aged 6 weeks were treated with Aldo injection ( $5 \mathrm{mg}$ per day, intraperitoneally) and/or oral administration of $2 \%$ salt water for 9 weeks. Control mice were treated with PBS injection (intraperitoneally) and/ or oral administration of distilled water for 9 weeks. (a) Systolic blood pressure and BW were continuously monitored. After indicated treatments for 3 weeks and 9 weeks, mice were killed and kidney weights were measured. (b) Excretion of 24-h urinary protein levels was also monitored for 9-week treatment. Results in all panels are shown as means \pm s.e.m. of data and the results were analyzed by analysis of variance.

a

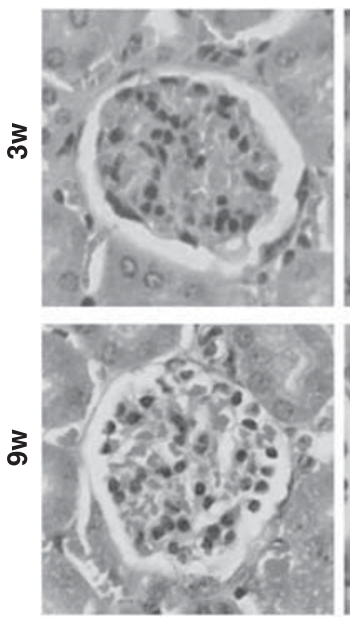

b
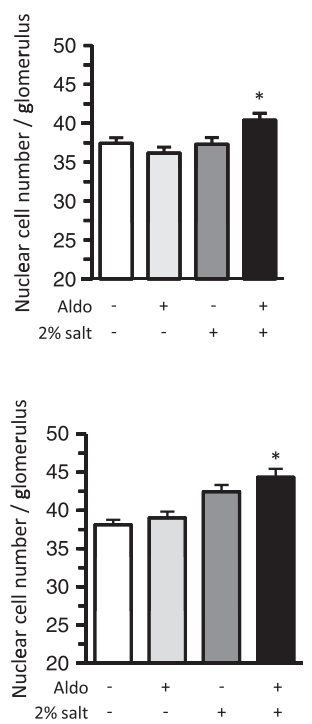

Aldo
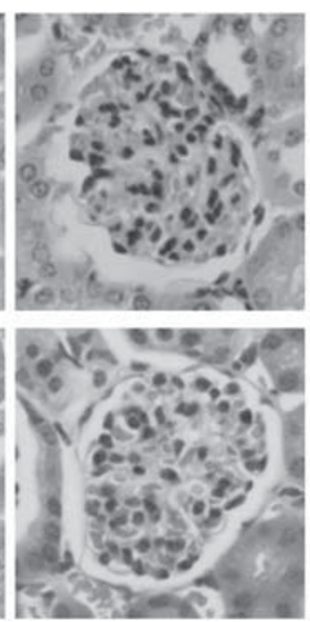

$2 \%$ Salt
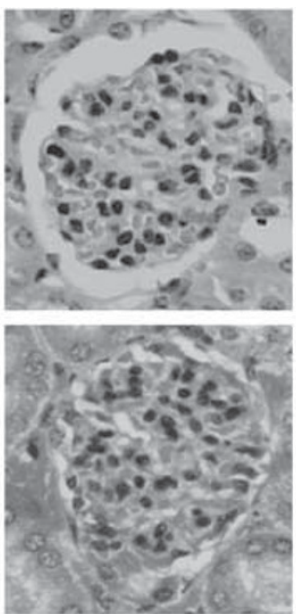

3 w-treatment

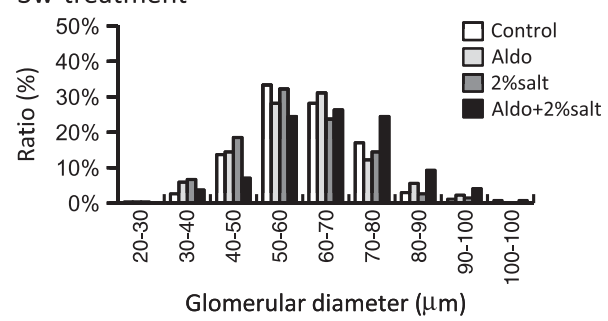

$9 w$-treatment

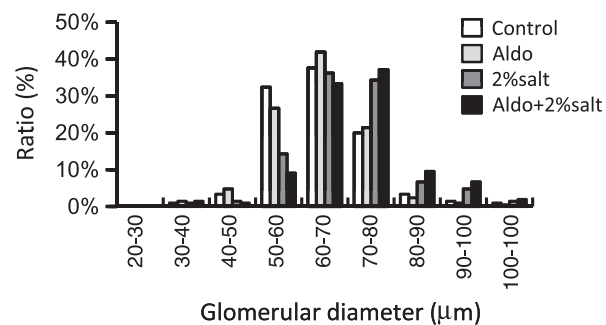

Figure 2 Histological changes in renal glomeruli treated with Aldo and high-salt intake. (a) For study of glomerular pathology, a portion of each kidney was sectioned and stained with hematoxylin-eosin. Each image is shown as $\times 200$ magnification. (b) The total number of cells containing nuclei in each glomerulus, at least 100 glomeruli in each specimen, was counted under a microscope, and measurement of the diameter of each of 200 randomly selected glomeruli was performed in each experimental group. Results in the left panel (b) are shown as means \pm s.e.m. of data and the results were analyzed by analysis of variance, and when a significant effect due to treatment was observed $(P<0.05)$, subsequent comparisons of group means were conducted using Fisher's protected least significant difference test. ${ }^{*} P<0.05$ vs. control groups. Results in the right panel (b) are shown as frequency distribution (\%) of each range of glomerular size diameter $(\mu \mathrm{m})$. A full color version of this figure is available at the Hypertension Research journal online. 
With regard to BMP receptors in the renal cortex, mRNA expression levels of BMP type-I receptors, ALK-2 and ALK-3, were not significantly changed by 3 -week and 9-week treatments with Aldo and high-salt administration (Figure 5). On the other hand, BMPR II mRNA level was increased by 3-week and 9-week treatments with Aldo injection and high-salt administration.

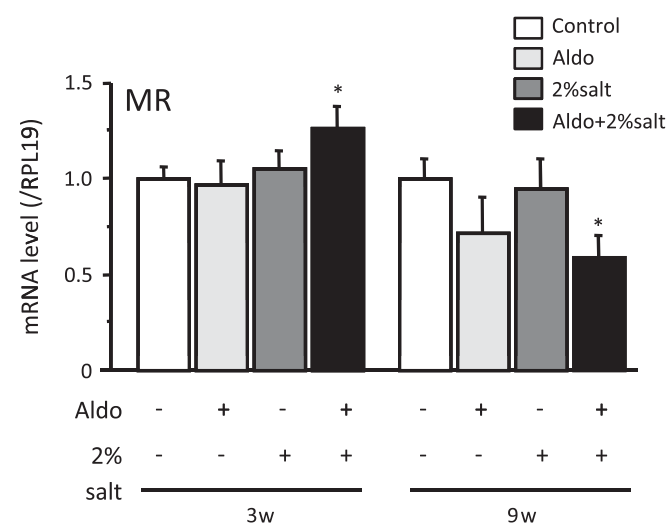

Figure 3 Effects of Aldo and high-salt administration on MR expression in the renal cortex. Total RNA was extracted from an adult male BALB/c mouse renal cortex, and the expression levels of MR mRNA were standardized by RPL19 level in each sample. Results are shown as means \pm s.e.m. of data from at least three separate experiments, each performed with triplicate samples. The results were analyzed by analysis of variance, and when a significant effect due to treatment was observed $(P<0.05)$, subsequent comparisons of group means were conducted using Fisher's protected least significant difference test. ${ }^{*} P<0.05 \mathrm{vs}$. control groups.

\section{DISCUSSION}

In the present study, we showed that expression of the renal BMP system is upregulated by treatment with Aldo and high-salt intake in vivo. Given that blood pressure did not change during the treatment with aldosterone and/or high-salt administration, mesangial expansion with increased cellularity induced by Aldo with high-salt intake possibly occurred by a direct mechanism via the MR.

The kidney exhibits a high density of MR expression along the tubular epithelium of the distal tubules and collecting ducts. ${ }^{5}$ The MR is also detected at glomeruli ${ }^{5}$ and preglomerular vasculature ${ }^{18}$ in the kidney. Aldo not only controls transport of water and electrolytes by stimulating MR but also causes renal injury in various hypertensive models. In animal hypertensive models, MR antagonism elicits renal protection independently of antihypertensive mechanisms. ${ }^{19}$ In the present study, MR mRNA level in the mouse renal cortex was increased by 3 -week treatment with Aldo and high-salt intake, suggesting that Aldo with high-salt exposure facilitates MR activities in the renal cortex at an early stage. At the delayed phase, MR expression was downregulated by the same treatment for 9 weeks. This phenomenon may imply the presence of autoregulatory feedback in the renal cortex exposed to a high concentration of Aldo. As BMP-4 and BMP-7 have inhibitory effects on MR expression in mesangial cells in vitro, ${ }^{15} \mathrm{MR}$ expression in the renal cortex could be secondarily downregulated by BMP effects activated by Aldo and high-salt administration.

BMP-4 and BMP-7 are important for the renal development. ${ }^{20}$ BMP-7 prevents metanephric mesenchymal cells from undergoing apoptosis and maintains them in the developmental arrest, ${ }^{21}$ while it promotes expansion of the stromal progenitor cells in the presence of a

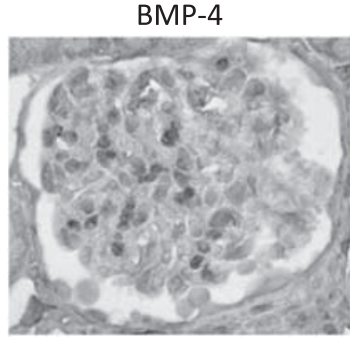

$9 w$ (Aldo+2\%salt)

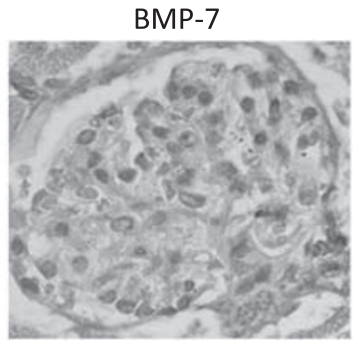

$9 w($ Aldo $+2 \%$ salt $)$

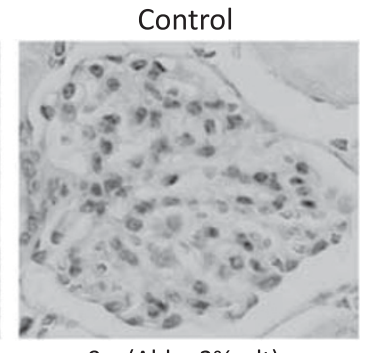

$9 \mathrm{w}$ (Aldo+2\%salt) b

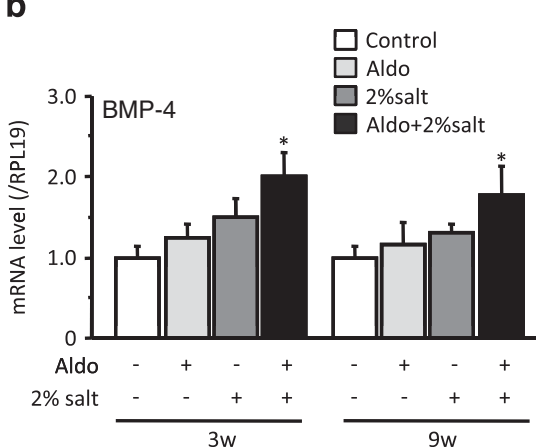

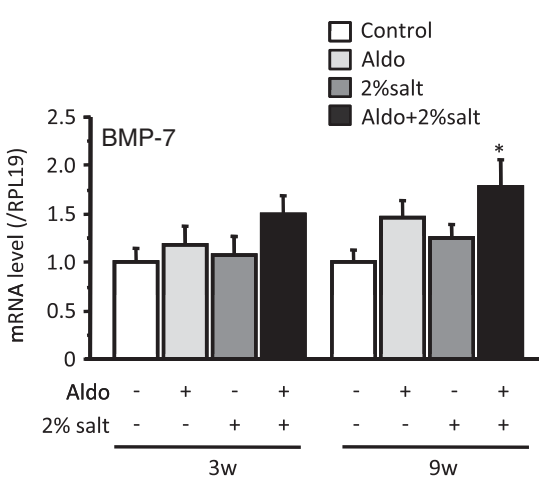

Figure 4 Effects of Aldo and high-salt administration on BMP-4 and BMP-7 expression in the renal cortex. (a) For immunohistochemical analyses, renal sections were reacted with goat polyclonal antibodies for BMP-4 and BMP-7 or a normal goat control antibody diluted at $15 \mu \mathrm{gml}-1$ for $16 \mathrm{~h}$ at $4{ }^{\circ} \mathrm{C}$ and were then stained using the universal immuno-peroxidase polymer method. Positive reaction was visualized with $3,3^{\prime}$-diaminobenzidine tetrahydrochloride, followed by counterstaining with hematoxylin and shown as $\times 400$ magnification. (b) Effects of Aldo and high-salt treatment on BMP-4 and BMP-7 expression in the renal cortex. Total RNA was extracted from an adult male BALB/c mouse renal cortex and the expression levels of BMP-4 and -7 mRNA were standardized by RPL19 level in each sample. Results in all panels are shown as means \pm s.e.m. of data from at least three separate experiments, each performed with triplicate samples. The results were analyzed by analysis of variance, and when a significant effect due to treatment was observed $(P<0.05)$, subsequent comparisons of group means were conducted using Fisher's protected least significant difference test. ${ }^{*}<0.05$ vs. control groups. A full color version of this figure is available at the Hypertension Research journal online. 

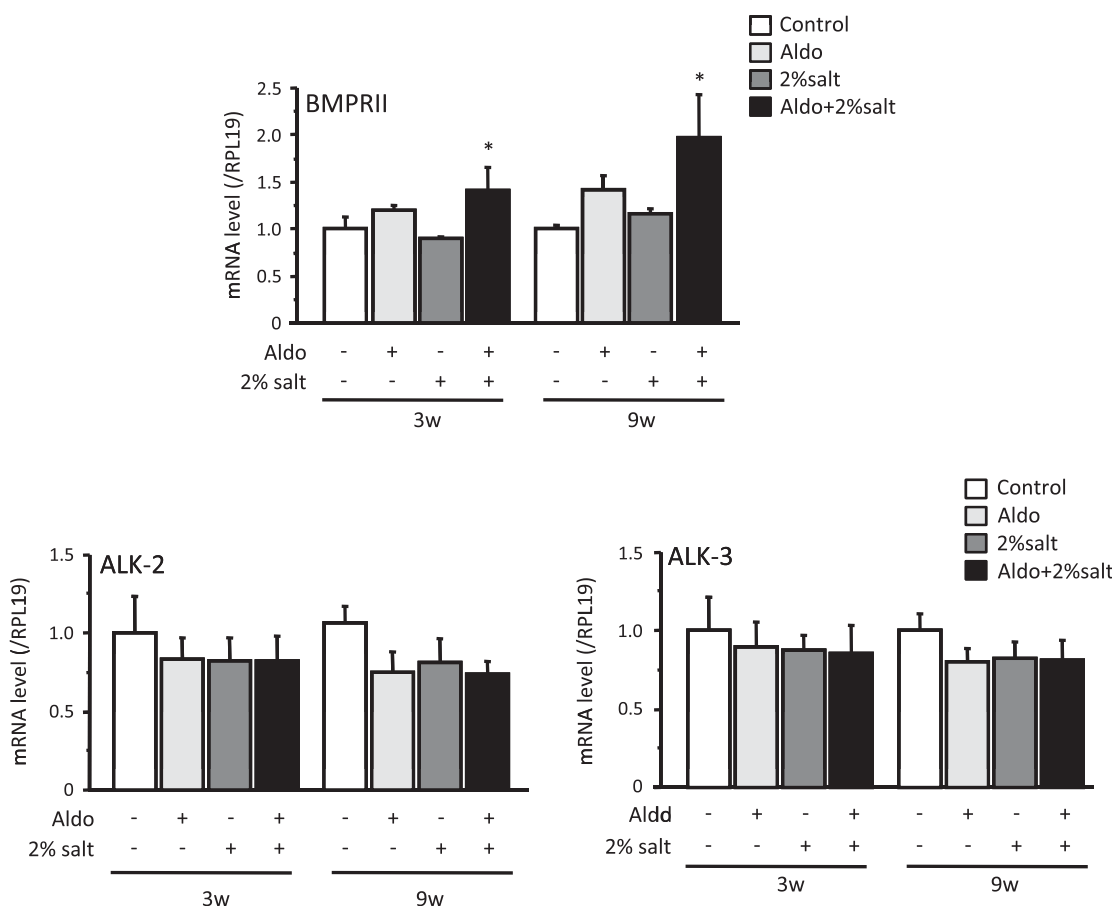

Figure 5 Effects of Aldo and high-salt administration on BMP receptor expression in the renal cortex. Total RNA was extracted from an adult male BALB/c mouse renal cortex, and the expression levels of BMP type II receptor, ALK-2 and ALK-3 mRNA were standardized by RPL19 level in each sample. Results in all panels are shown as means \pm s.e.m. of data from at least three separate experiments, each performed with triplicate samples. The results were analyzed by analysis of variance, and when a significant effect due to treatment was observed $(P<0.05)$, subsequent comparisons of group means were conducted using Fisher's protected least significant difference test. ${ }^{*} P<0.05$ vs. control groups.

fibroblast growth factor-2.22 BMP-4 inhibits the ureteric budding process but promotes the growth and elongation of the ureteric bud, leading to the branching morphogenesis of the kidney. ${ }^{23,24}$ In addition, BMP-7 reduces glomerular and tubulo-interstitial fibrosis and preserves renal function in experimental models of obstructive or diabetic nephropathy. ${ }^{12,25}$ BMP-7 treatment also resolves glomerular and interstitial fibrosis in rats with diabetic nephropathy, ${ }^{25}$ which results from inhibition of the TGF- $\beta$ effects on fibrosis mediators and matrix protein-degrading enzymes. ${ }^{13}$ In this regard, BMP-7 antagonizes TGF- $\beta$-induced glomerular fibrogenesis such as connective tissue growth factor expression and accumulation of collagen, fibronectin and thrombospondin in cultured mouse mesangial cells, suggesting that BMP-7 inhibits several profibrogenic activities of TGF- $\beta$ in mesangial cells. ${ }^{13}$ Renal TGF- $\beta$ expression is elevated in mineralocorticoid-salt hypertension ${ }^{26}$ or in Aldo-dependent remnant kidney model. ${ }^{27}$ Considering that the renal TGF- $\beta$ production is provoked by Aldo effects, ${ }^{27}$ BMPs may have an antagonistic role in Aldo-induced TGF- $\beta$ actions leading to amelioration of glomerular sclerosis.

Recently, Ueda et al. ${ }^{28}$ reported that BMP-4 dysfunction in developing podocytes leads to abnormal formation of glomerular capillary tuft. In that study, a BMP-binding protein, noggin-overexpressing mice in podocytes developed glomerular microaneurysms and collapse of glomerular capillary tufts. In contrast, BMP-4-overexpressing mice in podocyte developed glomeruli lacking both endothelial and mesangial cells. Their in vitro study also revealed that phosphorylated Smad signaling induced by BMP is present in endothelial and/or mesangial cells, but not in podocytes, within the developing glomeruli. These data suggest that podocyte-derived BMPs have an important role in glomerular capillary formation in a paracrine manner. ${ }^{28}$

Given that BMPs act to suppress mesangial cell proliferation in vitro, ${ }^{15}$ it is possible that the BMP system in the renal cortex has a role in antagonizing renal injury induced by Aldo and high-salt treatment. We previously reported that Aldo stimulated thymidine incorporation of mesangial cells in a concentration-dependent manner in vitro via MR actions. ${ }^{15}$ BMP-4 and BMP-7 suppressed basal and Aldo-induced mitotic activity. The antagonistic effects of BMP-4 and BMP-7 on mouse mesangial cell proliferation induced by Aldo were elicited by MR suppression as well as by inhibition of MR-induced MAPK activation. ${ }^{15}$ Taken together, BMP-4 and BMP-7 may protect the renal cortex tissue from Aldo and high-salt exposure.

In our earlier in vitro study, the expression levels of BMP-4 and BMP-7 mRNA were suppressed by treatment with Aldo in the mouse mesangial cell cultures. ${ }^{15}$ In the same culture condition of mesangial cells, MR expression was reduced by treatment with BMP-4 and BMP-7. As BMP-4 and BMP-7 antagonize Aldo-induced mesangial cell proliferation, it is possible that reduction of endogenous BMP-4 and BMP-7 is involved in increasing mesangial mitosis in the presence of Aldo. ${ }^{15}$ In the present in vivo study, we found that Aldo treatment with high-salt intake, but not Aldo alone, induced increased expression of BMP-4 and BMP-7 in the renal cortex tissue. Increased expression of BMP-4 and BMP-7 in the glomeruli could be induced by the glomerular stress such as glomerular hyperfiltration in the presence of both Aldo and high-salt loading. The upregulation of BMP-4 and BMP-7 may be involved in the reduction of MR expression shown in the expanded glomeruli treated with Aldo and high salt, although we cannot exclude the effects of contaminated tubular MR in this study using renal cortex tissues. Further study is needed to elucidate the molecular mechanism by which upregulation of BMP system occurs in renal tissues treated with Aldo and high-salt intake.

Collectively, it was uncovered that the BMP system in the renal cortex is activated by Aldo and high-salt treatment. Given that the renal BMP system acts to antagonize Aldo-induced mesangial 
proliferation, BMPs may have a key role in antagonizing glomerular damage induced by Aldo under the conditions of high-salt intake in vivo.

\section{CONFLICT OF INTEREST}

The authors declare no conflict of interest.

\section{ACKNOWLEDGEMENTS}

We thank Dr R Kelly Moore for helpful discussion and critical reading of the manuscript. We also appreciate the excellent technical assistance of Dr Hiroyuki Otani. This work was supported in part by Grants-in-Aid for Scientific Research, Daiichi-Sankyo Foundation of Life Science and Salt Science Research Foundation.

1 Rafiq K, Hitomi H, Nakano D, Nishiyama A. Pathophysiological roles of aldosterone and mineralocorticoid receptor in the kidney. J Pharmacol Sci 2011; 115: 1-7.

2 Nishiyama A, Hitomi H, Rahman A, Kiyomoto H. Drug discovery for overcoming chronic kidney disease (CKD): pharmacological effects of mineralocorticoid-receptor blockers. $J$ Pharmacol Sci 2009; 109: 1-6.

3 Nishiyama A, Yao L, Nagai Y, Miyata K, Yoshizumi M, Kagami S, Kondo S, Kiyomoto H, Shokoji T, Kimura S, Kohno M, Abe Y. Possible contributions of reactive oxygen species and mitogen-activated protein kinase to renal injury in aldosterone/salt-induced hypertensive rats. Hypertension 2004; 43: 841-848.

4 Nishiyama A, Yao L, Fan Y, Kyaw M, Kataoka N, Hashimoto K, Nagai Y, Nakamura E, Yoshizumi M, Shokoji T, Kimura S, Kiyomoto H, Tsujioka K, Kohno M, Tamaki T, Kajiya $\mathrm{F}$, Abe Y. Involvement of aldosterone and mineralocorticoid receptors in rat mesangial cell proliferation and deformability. Hypertension 2005; 45: 710-716.

5 Todd-Turla KM, Schnermann J, Fejes-Toth G, Naray-Fejes-Toth A, Smart A, Killen PD, Briggs JP. Distribution of mineralocorticoid and glucocorticoid receptor mRNA along the nephron. Am J Physiol 1993; 264: F781-F791.

6 Hostetter TH, Ibrahim HN. Aldosterone in chronic kidney and cardiac disease. J Am Soc Nephrol 2003; 14: 2395-2401.

7 Simic P, Vukicevic S. Bone morphogenetic proteins in development and homeostasis of kidney. Cytokine Growth Factor Rev 2005; 16: 299-308.

8 Wang SN, Lapage J, Hirschberg R. Loss of tubular bone morphogenetic protein-7 in diabetic nephropathy. J Am Soc Nephrol 2001; 12: 2392-2399.

9 Hruska KA, Guo G, Wozniak M, Martin D, Miller S, Liapis H, Loveday K, Klahr S, Sampath TK, Morrissey J. Osteogenic protein-1 prevents renal fibrogenesis associated with ureteral obstruction. Am J Physiol Renal Physiol 2000; 279: F130-F143.

10 Dudley AT, Lyons KM, Robertson EJ. A requirement for bone morphogenetic protein-7 during development of the mammalian kidney and eye. Genes Dev 1995; 9: 2795-2807.
11 Kitten AM, Kreisberg JI, Olson MS. Expression of osteogenic protein-1 mRNA in cultured kidney cells. J Cell Physiol 1999; 181: 410-415.

12 Morrissey J, Hruska K, Guo G, Wang S, Chen Q, Klahr S. Bone morphogenetic protein-7 improves renal fibrosis and accelerates the return of renal function. J Am Soc Nephrol 2002; 13(Suppl 1): S14-S21.

13 Wang S, Hirschberg R. BMP7 antagonizes TGF-beta -dependent fibrogenesis in mesangial cells. Am J Physiol Renal Physiol 2003; 284: F1006-F1013.

14 Dudley AT, Robertson EJ. Overlapping expression domains of bone morphogenetic protein family members potentially account for limited tissue defects in BMP7 deficient embryos. Dev Dyn 1997; 208: 349-362.

15 Otani H, Otsuka F, Inagaki K, Takeda M, Miyoshi T, Suzuki J, Mukai T, Ogura T, Makino $\mathrm{H}$. Antagonistic effects of bone morphogenetic protein- 4 and -7 on renal mesangial cell proliferation induced by aldosterone through MAPK activation. Am J Physiol Renal Physiol 2007; 292: F1513-F1525.

16 Otsuka F, Yamauchi T, Kataoka H, Mimura Y, Ogura T, Makino H. Effects of chronic inhibition of ACE and AT1 receptors on glomerular injury in dahl salt-sensitive rats. Am J Physiol 1998; 274: R1797-R1806.

17 Takeda M, Otsuka F, Suzuki J, Kishida M, Ogura T, Tamiya T, Makino H. Involvement of activin/BMP system in development of human pituitary gonadotropinomas and nonfunctioning adenomas. Biochem Biophys Res Commun 2003; 306: 812-818.

18 Uhrenholt TR, Schjerning J, Hansen PB, Norregaard R, Jensen BL, Sorensen GL, Skott $\mathrm{O}$. Rapid inhibition of vasoconstriction in renal afferent arterioles by aldosterone. Circ Res 2003; 93: 1258-1266.

19 Brown NJ. Aldosterone and end-organ damage. Curr Opin Nephrol Hypertens 2005; 14: 235-241.

20 Martinez G, Bertram JF. Organisation of bone morphogenetic proteins in renal development. Nephron Exp Nephrol 2003; 93: e18-e22.

21 Vukicevic S, Kopp JB, Luyten FP, Sampath TK. Induction of nephrogenic mesenchyme by osteogenic protein 1 (bone morphogenetic protein 7). Proc Natl Acad Sci USA 1996; 93: 9021-9026.

22 Dudley AT, Godin RE, Robertson EJ. Interaction between FGF and BMP signaling pathways regulates development of metanephric mesenchyme. Genes Dev 1999; 13: 1601-1613.

23 Lechner MS, Dressler GR. The molecular basis of embryonic kidney development. Mech Dev 1997; 62: 105-120.

24 Raatikainen-Ahokas A, Hytonen M, Tenhunen A, Sainio K, Sariola H. BMP-4 affects the differentiation of metanephric mesenchyme and reveals an early anterior-posterior axis of the embryonic kidney. Dev Dyn 2000; 217: 146-158.

25 Wang S, Chen Q, Simon TC, Strebeck F, Chaudhary L, Morrissey J, Liapis H, Klahr S, Hruska KA. Bone morphogenic protein-7 (BMP-7), a novel therapy for diabetic nephropathy. Kidney Int 2003; 63: 2037-2049.

26 Kim S, Ohta K, Hamaguchi A, Omura T, Yukimura T, Miura K, Inada Y, Wada T, Ishimura $Y$, Chatani F. Role of angiotensin II in renal injury of deoxycorticosterone acetate-salt hypertensive rats. Hypertension 1994; 24: 195-204.

27 Juknevicius I, Segal Y, Kren S, Lee R, Hostetter TH. Effect of aldosterone on renal transforming growth factor-beta. Am J Physiol Renal Physiol 2004; 286: F1059-F1062.

28 Ueda H, Miyazaki Y, Matsusaka T, Utsunomiya Y, Kawamura T, Hosoya T, Ichikawa I. $\mathrm{Bmp}$ in podocytes is essential for normal glomerular capillary formation. J Am Soc Nephrol 2008; 19: 685-694. 